\title{
Prevalence of polyps and endometrial cancer in a public hospital in Brazil
}

\section{RESENDE, LSA. Hospital de Base, Brasília -DF leandroresende@yahoo.com.br}

Intoduction: Endometrial polyps represent the localized growth of endometrial glands and stromal around a vessel, focal, which may occur anywhere in the uterine cavity. The proportion of glands, stromal and blood vessels will define their appearance. Most endometrial polyps are detected in asymptomatic women during imaging, especially transvaginal ultrasonography (TVS). However, their final diagnosis is histological and should be done on the specimen completely removed, usually by hysteroscopy. It is difficult to determine the exact prevalence of endometrial polyps in the female population without abnormal uterine bleeding (AUB). Many asymptomatic polyps are found on routine exams, especially TVS, hysterosonography, and hysteroscopy. In asymptomatic menopausal women, the prevalence of polyps varies from 13 to $16 \%$. However, the prevalence of polyps may reach more than $30 \%$ in pre and postmenopausal women with $\mathrm{AUB}^{4}$. The etiology of polyps remains controversial and the mechanisms involved in their development remain uncertain. As they are often positive for estrogen and progesterone receptors, it is possible that these hormones have a role in these pathology. Although genetic modifications have recently been extensively studied, the appearance of polyps seems to depend on the combination of several mutations. In addition, the penetrance of these genetic changes is possibly dependent on external factors such as metabolic, drug and environmental factors. Hysteroscopy-guided biopsy is currently the gold standard for the diagnosis of endometrial polyps because it has greater sensitivity and specificity. Hysteroscopy may be diagnostic or surgical. Diagnostic hysteroscopy only allows a subjective assessment of lesion characteristics, with low sensitivity and specificity, as compared to hysteroscopy resection.

Objectives: Classify the epidemiological profile of patients with endometrial pathologies treated in a public hospital in Brazil.

Methods: During two years (2016 - 2017) patients with endometrial pathologies were referred to the gynecologic oncology clinic of a public hospital in Brasilia - DF, Brazil. Patients were referred by presenting: endometrial thickening, bleeding in postmenopausal period, abnormal bleeding, and completed a clinical and epidemiological questionnaire. During the examination was drawn sample for biopsy when any abnormality was found.

\section{Results}

Table 1: distribution according to histology results

\begin{tabular}{cc}
\hline PATIENTS & $\mathbf{n}(\%)$ \\
\hline Total & $216(100)$ \\
Abnormalities & $60(27,8)$ \\
Polyps & $26(43,2)$ \\
Myoma & $4(6,7)$ \\
Simple hyperplasia & $12(20)$ \\
Simple atypical hyperplasia & $4(6,7)$ \\
Complex hyperplasia & $6(10)$ \\
Complex atypical hyperplasia & $4(6,7)$ \\
Adenocarcinoma & $4(6,7)$ \\
\hline
\end{tabular}

Table2: patient characteristics

\begin{tabular}{cr}
\hline \multicolumn{2}{c}{ Patients characteristics } \\
\hline Age & 52,3 years \\
BMI & $27,7 \mathrm{~kg} / \mathrm{m}^{2}$ \\
Endometrium thickness & $14,1 \mathrm{~mm}$ \\
\hline
\end{tabular}

Conclusions: According to National Data (INCA), endometrial cancer is the seventh most prevalent cancer. Brasilia - DF has a population of almost 3 million people and 418.000 women aged 40 years or older and only a hysteroscopy device available for the public health system. It has been estimated a total of 100 new cases of endometrial cancer in Brasília each year. This makes us think that there is a large number of undiagnosed cases, making the discovery of the disease is at an advanced stage, which increases the mortality and morbidity. Endometrial cancer is more prevalent in older women, in particular, over 50 years, which makes worse the picture is even more to know that the life expectancy of the Brazilian increased, significantly in recent decades. Among women it raises from 69.8 years in 1991 to 78.3 years in 2012 , almost 10 years. The availability in the public service of a greater number of hysteroscopy devices associated with endometrial biopsies could increase the number of patients diagnosed with endometrial cancer in women with symptoms or oligosymptomatic and, therefore, treatment in the initial phase of the disease presents better average survival results. 